\title{
Cardiac repair with pluripotent stem cell-derived cardiomyocytes: Proof of concept but new challenges
}

\author{
Hassan Masoudpour, MD, ${ }^{\mathrm{a}}$ and Michael A. Laflamme, $\mathrm{MD}, \mathrm{PhD}^{\mathrm{a}, \mathrm{b}}$
}

\footnotetext{
From the ${ }^{a}$ McEwen Centre for Regenerative Medicine, Toronto General Hospital Research Institute, University Health Network; and ${ }^{\mathrm{b}}$ Department of Laboratory Medicine and Pathobiology, University of Toronto, Toronto, Ontario, Canada.

M.L. received funding support from the McEwen Centre for Regenerative Medicine, the Toronto General Hospital Research Institute, the Ontario Institute for Regenerative Medicine, and the Medicine by Design/Canada First Research Excellence Fund.

Received for publication April 6, 2017; revisions received May 8, 2017; accepted for publication May 22, 2017; available ahead of print July 6, 2017

Address for reprints: Michael A. Laflamme, MD, PhD, University Health Network, 101 College St, Room 3-908, Toronto M5G 1L7, Ontario, Canada (E-mail: michael.laflamme@uhnresearch.ca).

J Thorac Cardiovasc Surg 2017;154:945-8

$0022-5223 / \$ 36.00$

Copyright $(2017$ by The American Association for Thoracic Surgery

http://dx.doi.org/10.1016/j.jtcvs.2017.05.088
}

Myocardial infarction (MI) remains among the leading causes of death in the United States and worldwide. Because the heart is a poorly regenerative organ, damaged myocardium is replaced by noncontractile scar tissue that reduces pump capacity and can initiate a process of structural and functional remodeling that often culminates in heart failure. Although there have been remarkable improvements in the medical management of post-MI patients in recent decades, whole-organ transplantation remains the only clinically available means of replacing lost contractile tissue and is the gold-standard treatment for end-stage heart failure. Unfortunately, the supply of donor hearts is inadequate to meet demand and those individuals fortunate enough to receive a heart transplant require close follow-up and lifelong immunosuppression, so many investigators have endeavored to develop cell-based therapies as an alternative strategy to remuscularize injured hearts and restore contractile function. Although the field initially focused on adult stem cells, including various bone marrow-derived cell populations, early clinical experience with these cell types has been generally disappointing, ${ }^{2}$ and there is now widespread consensus that they are incapable of differentiating into significant quantities of new cardiomyocytes (CMs). By contrast, multiple investigators have reported highly scalable methods for deriving phenotypically unambiguous CMs from pluripotent stem cells (PSCs). ${ }^{4}$ During the past decade, our group and others have shown that PSC-derived CMs can be used to regenerate infarcted hearts in multiple small animal models of MI. ${ }^{5-9}$ For example, our team has shown that CMs derived from pluripotent human embryonic stem cells (ESCs) can form stable grafts within the infarct region of small animal models and nonhuman primates and that the resultant graft myocardium is capable of electromechanical integration and 1:1 coupling with host tissue. ${ }^{7,8,10}$

The landmark discovery of induced PSCs (iPSCs) by Takahashi and colleagues ${ }^{11}$ provided an alternative source

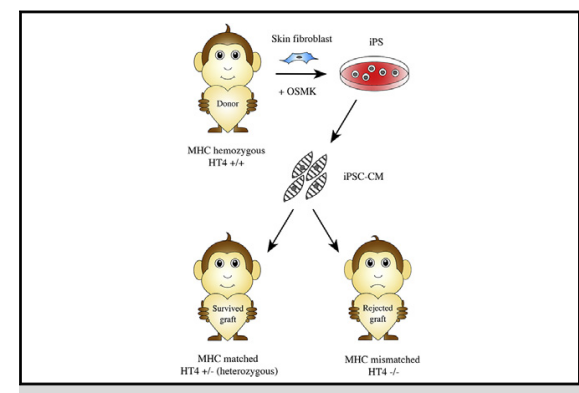

Schematic depicting experimental design and outcomes following induced pluripotent stem cell (PSC)-derived cardiomyocyte (CM) allotransplantation.

Central Message
Intracardiac injection of cardiomyocytes
derived from induced pluripotent stem cells
mediates beneficial effects on contractile func-
tion in a primate infarct model, but immuno-
logic and other issues must still be addressed.

See Editorial Commentary page 949.

of human PSCs that avoids many of the ethical and political objections associated with ESCs. iPSCs are created by reprogramming adult somatic cells into an ESC-like state via the forced expression of pluripotency-related transcription factors (the cocktail of Oct4, Sox2, c-Myc, and Klf4). Although this reprogramming event initially required gene delivery via integrating viral vectors, a number of potentially safer and even nonviral approaches have since been described, ${ }^{12}$ and these advances have significantly improved the prospects of iPSC-based therapies. Moreover, whereas ESC-based therapies would be allogeneic and require some degree of pharmacologic immunosuppression, iPSC technology could in principle enable autologous cell therapies where there is less risk of immunogenicity. By this strategy, one would create patient-derived iPSCs by reprogramming some readily accessible cell type (eg, dermal fibroblasts) and then guide their differentiation into some useful replacement cells (eg, CMs, neurons, and $\beta$-pancreatic cells). Toward this end, an important preclinical study recently reported in Nature by Shiba and colleagues ${ }^{13}$ provides exciting proof of concept for the use of iPSC-derived CMs (iPSC-CMs) in treating post-MI heart failure, although their work also highlights some significant and unexpected barriers to translation. 
In the study, Shiba and colleagues ${ }^{13}$ transplanted major histocompatibility complex (MHC)-matched primate iPSC-CMs in a nonhuman primate subacute MI model (cynomolgus monkeys) and showed that these CMs partially remuscularized the infarct scar and mediated robust improvements in contractile function. The resultant myocardial grafts survived for up to 3 months, occupied $\sim 16 \%$ of the infarct scar, and eventually became electrically coupled with host myocardium. To demonstrate their capacity for host-graft electrical coupling, the authors borrowed from an approach that had been previously used in transplantation studies with human ESC-CMs in small-animal and primate models ${ }^{7,8,10}$ and delivered cells that had been engineered to stably express a genetically encoded calcium-sensitive fluorescent protein (G-CaMP7.09). As was the case in the earlier work with GCaMP+ human ESC-CM grafts, the primate iPSC-CM grafts in this study exhibited fluorescent transients that occurred in 1:1 synchrony with the host echocardiogram, proving host-graft electromechanical integration. Also encouraging, none of the 5 infarcted monkeys that received MHC-matched iPSC-CMs showed teratoma formation, despite the implantation of 400 million cells per heart. The latter outcome supports earlier work with related PSC-CM populations, which emphasized the importance of high-purity $\mathrm{CM}$ populations before transplantation to avoid the risk of tumor formation. ${ }^{12}$

Although the preceding observations were largely confirmatory of earlier preclinical work with related PSC cardiac derivatives, ${ }^{5,7,8,10}$ the main novelty of this study is that it was the first to demonstrate that PSC-CM transplantation can mediate robust and apparently durable effects on left ventricle (LV) contractile function in a relevant largeanimal MI model. For this, Shiba and colleagues ${ }^{13}$ used high-resolution microcomputed tomography scanning and echocardiography to compare LV dimensions and contractile function in infarcted animals 2 days before thoracotomy and direct injection of either iPSC-CMs or vehicle alone (corresponding to 12 days post-MI) and at $4-$ and 12 -weeks posttransplantation. The authors did not include an experimental arm with infarcted monkeys undergoing sham or saline-alone injection, which is potentially important given that their hydrogel vehicle contains multiple well-known cardioprotective agents. Using high-resolution microcomputed tomography, they found that infarcted monkeys receiving iPSC-CMs exhibited statistically significant improvements in LV end-systolic volume and ejection fraction relative to infarcted vehicleonly recipients at both the 4- and 12-week time points. iPSC-CM recipients also showed a trend toward increased $\mathrm{LV}$ fractional shortening relative to vehicle controls by echocardiography, but this effect did not reach statistical significance, an outcome that the authors attributed to the reduced sensitivity of this imaging modality in postthoracotomy animals with significant postoperative adhesions. Although this explanation seems plausible, the study as a whole employed a small number of animals ( $n=5$ monkeys per group), so the authors were arguably fortunate to detect an effect by either imaging technique. Indeed, whereas this study by Shiba and colleagues ${ }^{13}$ represents exciting first proof of concept for the functional benefits of iPSC-CM transplantation in a large-animal model, follow-up experiments with larger group sizes and a longer duration of follow-up (3-6 months) seem definitely warranted.

Another important advance from this study by Shiba and colleagues ${ }^{13}$ is their description of a highly relevant model system for specifically addressing immunologic issues related to the allotransplantation of PSC-CMs. This is an area of tremendous uncertainty. Most prior work in the field has involved either the xenotransplantation of human PSC derivatives in animal models ${ }^{5,7-10}$ or the allotransplantation of mouse PSC derivatives in mice, ${ }^{14,15}$ approaches that have limited utility for predicting host immune responses following the allotransplantation of CMs in eventual human patients. Moreover, clinical experience with solid-organ transplantation may not extrapolate directly to outcomes following delivery of PSC-CMs alone in humans. In the latter case, the transplanted cell populations should be devoid of professional antigenpresenting cells present in whole hearts, such as endothelial cells. By comparison, CMs typically express low levels of MHC I antigens and undetectable levels of MHC II antigens. ${ }^{16}$

The system described by Shiba and colleagues ${ }^{13}$ is ideally suited to help resolve these uncertainties; determine the immunogenicity of PSC-CMs (whether syngeneic or from individuals with varying degrees of immunological mismatch); and explore strategies to overcome immune responses, including conventional immunosuppression or gene-edited universal donor PSCs. ${ }^{17,18}$ Although the authors have only begun to exploit the potential of their system (and their study involved only a small number of animals), their work has already yielded some insights that should inform the progress in the field. First, in a small pilot experiment, they transplanted iPSC-CMs into MHC-mismatched recipients and found that the graft cells were rejected by 1 month posttransplantation despite treatment with conventional immunosuppressant drugs (ie, tacrolimus and methylprednisolone). However, when the authors later transplanted these same donor cells into MHC-matched and similarly immunosuppressed recipients, grafts survived to the planned 3-month time point posttransplantation with no histologic evidence of cellular rejection. Because the authors did not test other immunosuppressant drugs or varying dosages, it remains to be determined precisely what level of immunosuppression would be required to prevent rejection of an MHC-matched iPSC-CM graft or 
if an alternative immunosuppressive regimen might have sufficed to prevent rejection of a mismatched graft.

In addition to the aforementioned concerns about graft cell rejection, the present study by Shiba and colleagues ${ }^{13}$ also highlighted another barrier to translation: The risk of graft-related arrhythmias. Infarcted monkeys receiving iPSC-CMs or vehicle alone underwent periodic Holter echocardiogram monitoring (on days $-2,+7,+14,+28$, $+42,+56,+70$, and +80 relative to thoracotomy and intracardiac delivery of iPS-CMs), and all cell recipients exhibited episodes of nonfatal sustained ventricular tachycardia (VT) that were not observed before cell transplantation or in vehicle controls. Despite worrisome bouts of VT up to $240 \mathrm{bpm}$ and multiple instances in which animals spent $100 \%$ of a 24 -hour monitoring period in VT, these tachyarrhythmias were reportedly well tolerated with no abnormal behavior, syncope episodes, or mortality. The authors reported an interesting time course for these arrhythmias with a peak incidence at $\sim 14$ days posttransplantation, followed by a gradual reduction over time (with the incidence of VT declining from $100 \%$ of cell recipients at 14 days to $20 \%$ by 80 days posttransplantation). Because the morphology, incidence, and time course of these graft-related tachyarrhythmias are qualitatively similar to those previously reported by Chong and colleagues $^{10}$ following the transplantation of human ESC-CMs in another nonhuman primate MI model, VT seems likely to be a generalizable phenomenon that can be expected following any significant remuscularization with PSC-CMs in large animals. Importantly, these graft-related arrhythmias were not predicted by earlier PSC-CM transplantation studies in small-animal models. ${ }^{7,8}$ This difference may reflect the suppression of graft cell ectopy by the faster sinus rate of rodent recipients and/or their smaller heart and graft sizes with shorter paths of conduction for re-entrant arrhythmias to arise. To some extent, these same limitations might also apply to the primate model employed by Shiba and colleagues ${ }^{13}$ because these were relatively small, rabbit-sized monkeys (with hearts weighing $\sim 10 \mathrm{~g}$ and a sinus rate of $\sim 160 \mathrm{bpm}) .{ }^{19,20}$

\section{NEXT STEPS}

Shiba and colleagues ${ }^{13}$ have provided exciting first proof of concept for the use of PSC-CMs in a large-animal MI model, and they have described a highly relevant platform that should be very useful for addressing immunologic issues with these cells. However, as with any study, there are important limitations to their work that will need to be addressed in future experiments if this therapeutic approach is to advance to clinical use. First and foremost, the field needs to determine the mechanistic basis of tachyarrhythmias observed following iPSC-CM transplantation and define an effective strategy to eliminate them. Even if these graft-related arrhythmias are transient in nature, their occurrence in a patient population already at elevated risk of arrhythmias is obviously unacceptable. As noted above, the small primates in the study by Shiba and colleagues ${ }^{13}$ have a rapid sinus rate that significantly exceeds that of humans and so may mask graft-related arrhythmias that may otherwise occur in patients, so transplantation studies in a larger, slower-rated preclinical (eg, swine or canine) model may be more informative. Second, whereas this study represents the first demonstration of efficacy with iPSC-CMs in a large-animal model, it will be important to re-examine the functional consequences of iPSC-CM transplantation in a follow-up study involving a larger cohort of animals and a longer duration of follow-up. Indeed, other cell therapies have shown transient beneficial effects that have not persisted to later time points. ${ }^{21}$ Third, as noted above, the authors have described an elegant system for investigating the immunobiology of PSC-CM allografts, but additional experiments will be required to better define the immunogenicity of these cells and to determine the most appropriate immunosuppression regimen with any given degree of MHC mismatch. It would also be useful to know the fate of iPSC-CM grafts in MHCmatched recipients in the absence of immunosuppression. The authors imply that MHC-matched cells would be rejected, but that was never directly tested in the present study. Work to address these immunologic issues would probably be better performed using wild-type cells, rather than iPSC-CMs that have been engineered to express GCaMP, as in the present study. GCaMP is a potential confounder: It is a derivative of the jellyfish green fluorescent protein, which is known to be highly immunogenic. ${ }^{22}$

Finally, a common limitation of not only this report but also nearly all prior work in the cardiac cell therapy field, is that we have incomplete mechanistic insights into how the transplantation of these cells actually mediate beneficial effects on LV contractile function. Indeed, Shiba and colleagues $^{13}$ did show that their iPSC-CM grafts are capable of electromechanical integration and 1:1 coupling with host myocardium, but this finding does not prove that the salutary effects of iPSC-CM transplantation are direct and result from the formation of new, synchronously activated force-generating units. Other indirect mechanisms cannot be ruled out, including passive mechanical buttressing by the large myocardial implants, reduced wall thinning, activation of endogenous reparative mechanisms, and/or paracrine release of proangiogenic and/or cardioprotective factors. ${ }^{23}$ (The latter mechanism, the paracrine release of factors that promote the salvage of host CMs, has been reported to be particularly important in mediating the beneficial effects of other candidate cell therapies. ${ }^{24}$ ) Going forward, Shiba and colleagues ${ }^{13}$ may be able to address some of these potential mechanisms using straightforward histologic end points, perhaps even on existing materials 
(eg, by looking for evidence of increased border zone capillary density or reduced host cell death). Elucidating other potential mechanisms may be more challenging and may even require creation of new tools to better reveal how the graft CMs are operational. Nonetheless, this is a critical issue to resolve that has large implications for translational issues such as the most appropriate timing, dose, and route of cell administration. Shiba and colleagues $^{13}$ have provided compelling evidence that iPSC-CMs can improve contractile function in infarcted hearts, so a critical next step will be to determine precisely how these cells mediate this effect.

\section{Conflict of Interest Statement}

Dr Laflamme is a founding investigator, equity holder, and a paid consultant for BlueRock Therapeutics Corp. Dr Masoudpour has nothing to disclose with regard to commercial support.

\section{References}

1. Benjamin EJ, Blaha MJ, Chiuve SE, Cushman M, Das SR, Deo R, et al. Heart Disease and Stroke Statistics-2017 update: a report from the American Heart Association. Circulation. 2017;135:e146-603.

2. Gyongyosi M, Wojakowski W, Lemarchand P, Lunde K, Tendera M, Bartunek J, et al. Meta-Analysis of Cell-based CaRdiac stUdiEs (ACCRUE) in patients with acute myocardial infarction based on individual patient data. Circ Res. 2015;116: 1346-60.

3. Laflamme MA, Murry CE. Heart regeneration. Nature. 2011;473:326-35.

4. Hartman ME, Dai DF, Laflamme MA. Human pluripotent stem cells: prospects and challenges as a source of cardiomyocytes for in vitro modeling and cellbased cardiac repair. Adv Drug Deliv Rev. 2016;96:3-17.

5. van Laake LW, Passier R, Doevendans PA, Mummery CL. Human embryonic stem cell-derived cardiomyocytes and cardiac repair in rodents. Circ Res. 2008;102:1008-10.

6. Caspi O, Huber I, Kehat I, Habib M, Arbel G, Gepstein A, et al. Transplantation of human embryonic stem cell-derived cardiomyocytes improves myocardial performance in infarcted rat hearts. J Am Coll Cardiol. 2007;50:1884-93.

7. Shiba Y, Filice D, Fernandes S, Minami E, Dupras SK, Biber BV, et al. Electrical integration of human embryonic stem cell-derived cardiomyocytes in a guinea pig chronic infarct model. J Cardiovasc Pharmacol Ther. 2014;19:368-81.
8. Shiba Y, Fernandes S, Zhu WZ, Filice D, Muskheli V, Kim J, et al. Human EScell-derived cardiomyocytes electrically couple and suppress arrhythmias in injured hearts. Nature. 2012;489:322-5.

9. Laflamme MA, Chen KY, Naumova AV, Muskheli V, Fugate JA, Dupras SK, et al. Cardiomyocytes derived from human embryonic stem cells in pro-survival factors enhance function of infarcted rat hearts. Nat Biotechnol. 2007;25:1015-24.

10. Chong JJ, Yang X, Don CW, Minami E, Liu YW, Weyers JJ, et al. Human embryonic-stem-cell-derived cardiomyocytes regenerate non-human primate hearts. Nature. 2014;510:273-7.

11. Takahashi K, Tanabe K, Ohnuki M, Narita M, Ichisaka T, Tomoda K, et al. Induction of pluripotent stem cells from adult human fibroblasts by defined factors. Cell. 2007;131:861-72.

12. Singh VK, Kumar N, Kalsan M, Saini A, Chandra R. Mechanism of Induction: induced pluripotent stem cells (iPSCs). J Stem Cells. 2015;10:43-62.

13. Shiba Y, Gomibuchi T, Seto T, Wada Y, Ichimura H, Tanaka Y, et al. Allogeneic transplantation of iPS cell-derived cardiomyocytes regenerates primate hearts. Nature. 2016;538:388-91.

14. Liao SY, Liu Y, Siu CW, Zhang Y, Lai WH, Au KW, et al. Proarrhythmic risk of embryonic stem cell-derived cardiomyocyte transplantation in infarcted myocardium. Heart Rhythm. 2010;7:1852-9.

15. Klug MG, Soonpaa MH, Koh GY, Field LJ. Genetically selected cardiomyocytes from differentiating embronic stem cells form stable intracardiac grafts. J Clin Invest. 1996;98:216-24.

16. Karabekian Z, Posnack NG, Sarvazyan N. Immunological barriers to stem-cell based cardiac repair. Stem Cell Rev. 2011;7:315-25.

17. Riolobos L, Hirata RK, Turtle CJ, Wang PR, Gornalusse GG, Zavajlevski M, et al. HLA engineering of human pluripotent stem cells. Mol Ther. 2013;21: 1232-41.

18. Wang D, Quan Y, Yan Q, Morales JE, Wetsel RA. Targeted disruption of the beta2-microglobulin gene minimizes the immunogenicity of human embryonic stem cells. Stem Cells Transl Med. 2015;4:1234-45.

19. Keenan CM, Vidal JD. Standard morphologic evaluation of the heart in the laboratory dog and monkey. Toxicol Pathol. 2006;34:67-74.

20. Gauvin DV, Tilley LP, Smith FW Jr, Baird TJ. Electrocardiogram, hemodynamics, and core body temperatures of the normal freely moving cynomolgus monkey by remote radiotelemetry. J Pharmacol Toxicol Methods. 2006;53: 140-51.

21. Nguyen PK, Rhee JW, Wu JC. Adult stem cell therapy and heart failure, 2000 to 2016: a systematic review. JAMA Cardiol. 2016;1:831-41.

22. Ansari AM, Ahmed AK, Matsangos AE, Lay F, Born LJ, Marti G, et al. Cellular GFP toxicity and immunogenicity: potential confounders in in vivo cell tracking experiments. Stem Cell Rev. 2016;12:553-9.

23. Laflamme MA, Zbinden S, Epstein SE, Murry CE. Cell-based therapy for myocardial ischemia and infarction: pathophysiological mechanisms. Annu Rev Pathol. 2007;2:307-39.

24. Hodgkinson CP, Bareja A, Gomez JA, Dzau VJ. Emerging concepts in paracrine mechanisms in regenerative cardiovascular medicine and biology. Circ Res. 2016;118:95-107. 